\title{
An ETBG Optimization Algorithm Based on Analytic Hierarchy Process in WSN
}

\author{
Xin-fang Yan ${ }^{1,2}$, Yong-kun Zhang ${ }^{2}$, Hai-ling Tang ${ }^{1}$, Shao-nong $\mathrm{Li}^{2}$ \\ ${ }^{1}$ School of Information Engineering, Huang He Science and Technology College, Zhengzhou, China \\ ${ }^{2}$ School of Information Engineering, Zhengzhou University, Zhengzhou, China
} 15896515961@163.com

\begin{abstract}
To solve the problems which exist in Energy-aware Topology Control Protocol Based on Gradient(ETBG) algorithm, one strategy that considers a combination of factors to choose the cluster head is proposed based on Analytic Hierarchy Process(AHP). This way not only uses the comprehensive weight that composed of the residual energy, number of neighbor nodes, average distance to the neighbor nodes, distance to the center of gradient, but also utilizes mathematical methods to determine weight coefficients, to reduce the interference of human factors and make the elected nodes enough optimized to serve as cluster heads. Simulation results show that the elected cluster heads distribute more properly in space, so that the network lifetime can be further extended and the algorithm is suitable for large-scale sensor network.
\end{abstract}

Keywords-WSN; ETBG; cluster head choosing; weight coefficients; AHP;

\section{INTRODUCTION}

After the computer and network, Internet of things (IOT) has become the third wave of world information sector. In recent years, as the Internet of things has attracted more and more attention, wireless sensor network (WSN) has entered a period of rapid development. It has many characteristics like the big scale, self-organization and so on. However, after node disposable spread, the node energy is usually nonrenewable. Therefore, reduce network energy consumption and prolong network lifetime become the primary sensor network designed objective by the agreement [1, 2]. Clustering routing algorithm has been extensively studied and applied thanks to its expanding energy efficiency and easy maintenance [3-6].

Literature [6] introduces a clustering algorithm based on energy (ETBG), the algorithm refer to the gradient of directed diffusion protocol, build the network into a gradient field according to the node's communication radius to reduce the grade cluster level and data packet forwarding hops, reduce latency and at the same time with the base station for a specific mobile network to extend the life cycle.

However, because of that this algorithm only considers the remaining strategic energy in cluster head selection without taking into account the distance, position and other factors, some nodes are not fit and die for excessive energy consumption. To solve this problem, one improved strategy to choose cluster heads based on the idea of Analytic Hierarchy Process is proposed, so that the network lifetime can be further extended, the algorithm is suitable for largescale sensor network.

\section{ENERGYPE MODEL}

We use node transceiver energy model in literature [3], according to this model, energy consumption by transmitting data is proportional to the transmission distance $n(n \geq 2)$, and the greater the distance, the greater the $\mathrm{n}$ values; In this model, when a sensor node transmit a k-bit message in a distance $\mathrm{d}$ to another one, the energy consumption of transceiver for sending the message is:

$$
\begin{array}{ll}
E_{T X}(k, d)=E_{\text {elec }} \times k+\varepsilon_{\text {amp }} \times k \times d^{2} & \left(d \leq d_{0}\right) \\
E_{T X}(k, d)=E_{\text {elec }} \times k+\varepsilon_{\text {amp }} \times k \times d^{4} & \left(d>d_{0}\right)
\end{array}
$$

The energy consumption for receiving the message is:

$$
E_{R X}(k)=E_{R X_{-} \text {elec }}(k)=E_{\text {elec }} \times k \quad\left(d>d_{0}\right)
$$

Among them, $E_{\text {elec }}=50 \mathrm{~nJ} / \mathrm{bit}$ show the energy consumption of transceiver for transmitting and receiving one bit of message, $\varepsilon_{a m p}=100 \mathrm{pJ} / \mathrm{bit} / \mathrm{m}^{2}$ and $\varepsilon_{a m p}=100 \mathrm{pJ} / \mathrm{bit} / \mathrm{m}^{4}$ indicate the coefficient factor of energy consumption or transmitting one bit message over $\mathrm{d}$ meter, critical distance $\mathrm{d} 0=87.7 \mathrm{~m}$, length of message packet $k=128 \mathrm{bit}$.

\section{OPTIMIZATION AlgORITHM}

\section{A. The Main Idea of Optimization}

In clustering routing protocol, the main idea is that cluster heads fuse the data which received by their cluster members and then send to BS. So cluster heads have two tasks: data fusion and transmission. Thus, cluster heads consume more energy than other member nodes and it is very important to choose ideal ones.

An optimization algorithm is proposed based on AHP in this paper. It considers a combination of four factors to choose the cluster head, the residual energy, number of neighbor nodes, average distance to the neighbor nodes and distance to the center of gradient. So comprehensive weight value is:

$$
W_{\mathrm{i}}=w_{1} \times E_{r}+w_{2} / N_{d}+w_{3} / D_{a}+w_{4} / D_{m} \text { (4) }
$$

$E_{r}$ : The first weight component is residual energy. The node which has more residual energy is more likely to be selected as cluster head;

$N_{d}$ : The second component is absolute value of the difference of the number of neighbor nodes and optimal neighbor nodes. The more the number of node neighbors close to the optimal number of neighbor nodes, this cluster is more close to the optimal cluster; 
$D_{a}$ : The third component is average distance to the neighbor nodes. Assumed that the network node is connected when the node transmitting radius is $\mathrm{d}$, then it is defined:

$$
D_{\mathrm{a}}=\sum_{i=1}^{m} \frac{\text { Distonbr }}{m}
$$

$D_{m}$ : The fourth component is distance to the center of gradient. The node which is near to the center of gradient energy is more likely to be selected as cluster head;

$w_{i}(i=1,2,3,4)$ : They are the weight coefficients. The methods to determine weight coefficient will be introduced in the next section.

\section{B. Analytic Hierarchy Process}

Starting from the global system, we build a model to analyze and determine the relative important degree of the four factors by Analytic Hierarchy Process.

AHP is a multiple criteria decision-making method. It decomposes a complex problem into a hierarchy of simple sub problems (or factors), synthesizes their importance to the problem, and finds the best solution. In this paper, AHP is used to deal with cluster head selection and is carried out in three steps:

Step1: Collect information and structure the cluster head selection problem as a decision hierarchy of independent factors.

Step2: Calculate the relative local weights of decision actors or alternatives of each level. The evaluation matrices are built up through pairwise comparing each decision factor under the topmost goal. The comparison results which are implemented by asking the questions: "Which is more important? How much?" are presented in square matrix A, in which $a_{i j}$ denotes the ratio of the $i$ th factor weight to the $j$ th factor weight. The fundamental 1 to 9 scale can be used to rank the judgments as shown in Table 1.

TABLE I. A FundAMENTAL 1 TO 9 SCALE

\begin{tabular}{cc}
\hline Number Rating & Verbal Judgment of Preferences \\
\hline 1 & Equally preferred \\
3 & Moderately preferred \\
5 & Strongly preferred \\
7 & Very strongly preferred \\
9 & Extremely preferred \\
\hline
\end{tabular}

$2,4,6,8$ indicate the medium value of above pairwise comparison.

Step3: For the matrix A, we calculate its eigenvalue equation written as $\mathrm{AW}=\lambda_{\max } \mathrm{W}$, where $\mathrm{W}$ is non-zero vector called eigenvector, and $\lambda_{\max }$ is a scalar called eigenvalue.

If every element satisfies the equations and, the matrix A is the consistency matrix. Unfortunately, the evaluation matrices are often not perfectly consistent due to people's random judgments. These judgment errors can be detected by a consistency radio (CR), which is defined as the radio of consistency index (CI) to random index (RI). CI can be achieved by

$$
\lambda_{\max }=\sum_{j=1}^{n} \frac{(A W)_{j}}{n c_{j}}
$$

And RI is shown in Table 2. When $\mathrm{CI} \leqslant 0.01$, the judgment errors are tolerable and the weight coefficients of the global weight matrix is the weights of decision factor under the topmost goal. Otherwise, the pairwise comparisons should be adjusted until matrix A satisfies the consistency check.

\section{An ETBG Optimization Algorithm Based on AHP}

When $\mathrm{CI} \leqslant 0.01$, the judgment errors are tolerable and the weight coefficients of the global weight matrix is the weights of decision factor under the topmost goal. Otherwise, the pairwise comparisons should be adjusted until matrix A satisfies the consistency check.

In the gradient building phase, Base Station still take $\mathrm{nR}$ as Communication radius in turn and sent massage of gradient building $(n=1, \ldots,[D / R],[D / R]$ is an integer and $\mathrm{D} / \mathrm{R} \leqslant[\mathrm{D} / \mathrm{R}]<\mathrm{D} / \mathrm{R}+1$ ). Nodes in the network will set their own level as $\mathrm{n}$ after they received the message and then calculate the distance to the center of its own gradient.

When forming the first Cluster, each node in the Network sends message in one Step Neighbor to exchange the information of neighbor nodes (Residual energy, number of neighbor nodes, average distance to the neighbor nodes, distance to the center of gradient). Any node v compute the comprehensive weights by using (4);

We can calculate the value of $w_{i}(i=1,2,3,4)$ by adopting the methods in last section B.

a. Structuring a hierarchical model;

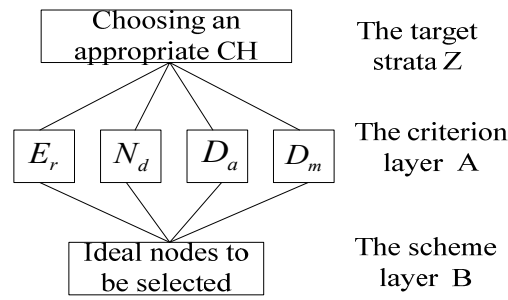

Figure 1. Hierarchical model.

The goal of the decision "choosing an appropriate $\mathrm{CH}$ " is at the top level of the hierarchy as shown in Fig. 1. The next level consists of the decision factors which are called criteria for this goal. And at the bottom level are the alternative sensor nodes to be evaluated.

b. Making pairwise comparison

We can construct a judgment matrix $\mathrm{A}$ as shown below according to the methods in last section B.

$$
A=\left\{\begin{array}{cccc}
1 & 3 & 5 & 8 \\
\frac{1}{3} & 1 & 2 & 5 \\
\frac{1}{5} & \frac{1}{2} & 1 & 3 \\
\frac{1}{8} & \frac{1}{5} & \frac{1}{3} & 1
\end{array}\right\}
$$

Then $\lambda_{\max }=4.0540$;

c. Making pairwise comparison.

CI can be achieved by

$$
C I=\frac{\lambda_{\max }-n}{n-1}=\frac{4.0540-4}{4-1}=0.0018(n=4)
$$

$\mathrm{RI}$ is shown in Table II; 
TABLE II. CONSISTENCY INDEX

\begin{tabular}{|c|c|c|c|c|c|c|c|}
\hline Dimension & $\mathbf{1 , 2}$ & $\mathbf{3}$ & $\mathbf{4}$ & $\mathbf{5}$ & $\mathbf{6}$ & $\mathbf{7}$ & $\boldsymbol{\cdots}$ \\
\hline $\mathrm{RI}$ & 0 & 0.58 & 0.90 & 1.12 & 1.24 & 1.32 & $\ldots$ \\
\hline
\end{tabular}

As shown in table $2, \mathrm{RI}=0.9$ when $\mathrm{n}=4$. So the value of Consistency ratio is: $\mathrm{CR}=\mathrm{CI} / \mathrm{RI}=0.002<0.01$

So matrix A satisfies the consistency check. Then we can calculate weighting coefficients of four facts by using:

$$
\begin{aligned}
& \lambda_{\max }=\sum_{j=1}^{4} \mathrm{a}_{i j} / \sum_{i, j=1}^{4} \mathrm{a}_{i j} \\
& w_{1}=0.5364, w_{2}=0.2630, w_{3}=0.1483, w_{4}=0.0523
\end{aligned}
$$

Through the above steps, each node with the same level in the network will form a cluster which makes the node that have the largest weights as the cluster head.

After optimized of cluster heads, cluster tree is generated according to ETBG.

\section{An Example}

Using the energy model described in [3], we run simulations to evaluate the performance of this algorithm. Assuming that 100 and 500 nodes are randomly distributed in a $100 * 100$ area, each node is initially given $0.5 \mathrm{~J}$ of energy and the maximal communication radius $R$ is $40 \mathrm{~m}$. The BS is not energy-constrained and located at $(50,0)$. We assume that the radio dissipates $E_{\text {elec }}=50 \mathrm{~nJ} / \mathrm{bit}$ to run the transmitter and receiver circuitry and $\varepsilon=10 p J$ for the transmit amplifier to transmit a bit of information for one meter. The length of the news packet is $1=128 \mathrm{bit} /$ packet and energy consumption for data fusion is $E_{D A}=5 \mathrm{~nJ} / \mathrm{bit}$.

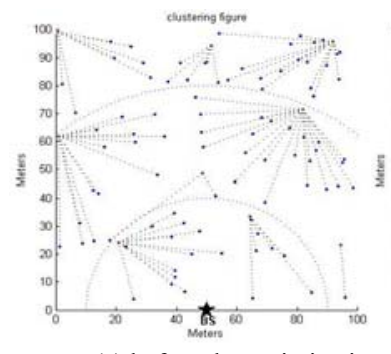

(a) before the optimization

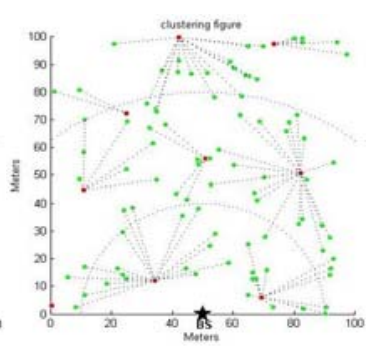

(b) after the optimization
The dividing clusters figure of 100 nodes

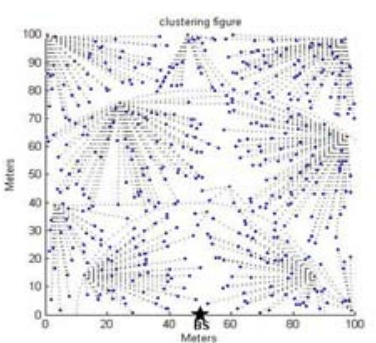

(a) before the optimization

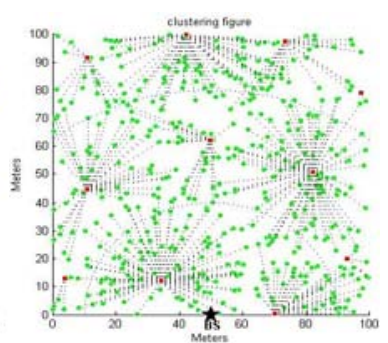

(b) after the optimization
The dividing clusters figure of 500 nodes

Figure 2. Before and after algorithm optimization compared with different dividing clusters

We can see from the comparison of Fig. 2: selected cluster head before optimization is far from the gradient centerline, and close to the edge of the cluster; after optimization, because of the using of the comprehensive weights based on the hierarchical analysis, selected cluster head is closer to the gradient centerline, and more closer to the of cluster, so that it can reduce the total energy consumption of the intra-cluster communication greatly and extend the survival period of the network.

\section{SimUlation AND ANALYSIS}

\section{A. Different densities od nodes}

As the cluster heads' energy consumption accounts for main part of the energy consumption in the network, we compare the total energy which the cluster heads consume before and after optimization when there are different number of nodes. The number of nodes selected in the experiment are 20,40,.., 200, the result is shown in Fig. 3.

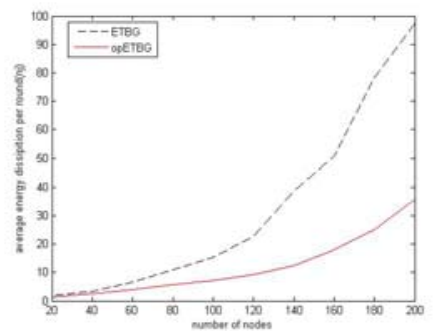

Figure 3. Consuming energy of cluster head vs. Number of nodes

It is concluded in Fig. 3 that as the number of nodes in the area is increasing, the number of nodes around the cluster heads is becoming more and more intensive. Responding, the task of the cluster heads is more onerous and the energy consumption is increasing. After the optimization algorithm, the cluster heads selected are more close to gradient centerline and centroid of the cluster, so that it could reduce the total energy which the cluster heads communication consumed to the maximum extent.

\section{B. Contract on Lifetime}

Fig. 4 shows a comparison of system lifetime using opETBG versus ETBG, both utilizing periodic rotation of cluster-heads. It can be seen that, when the number of the rounds after each time of clustering increases from 1 to 2200 , op-ETBG maintains a relatively stable high-value compared to ETBG. This indicates that, as long as the frequency of periodic rotation of cluster-heads is controlled within this range, op-ETBG will enable long system lifetime compared to ETBG. But when the number of the rounds after each time of clustering exceeds 3500, op-ETBG and ETBG both show a downward trend obviously. Thus it can be seen that, for wireless sensor networks, whose main function is data transmission, to choose a suitable time for periodic rotation of cluster-heads plays an important role in enhancing network lifetime. 


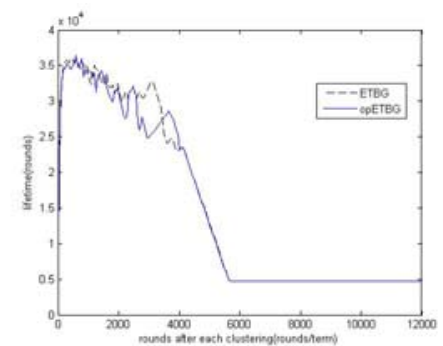

Figure 4. Lifetime vs. Frequency of cluster-heads rotation

\section{Various Communication Radius}

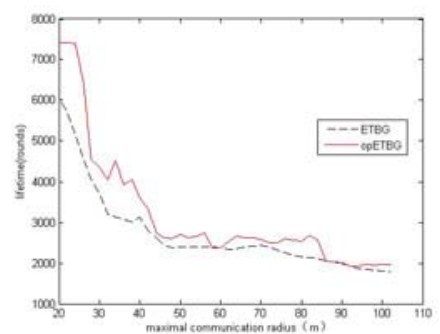

Figure 5. Lifetime vs. Communication radius

Fig. 5 shows a comparison of system lifetime using ETBG versus op-ETBG as the communication radius increases. If the communication radius of the node is too small, the network would be unconnected (It is $20 \mathrm{~m}$ in the simulation). From $20 m$ to $35 m$, op-ETBG performs better than ETBG does. After that, the number of the rounds for data transmission decreases gradually as the communication radius increases because the energy expended in exchanging information in clustering phase will be increased inevitably while d increases..

\section{CONCLUSION}

This text puts forward a optimization algorithm of ETBG. It balances the load of each cluster head in the network by using comprehensive weight value composed of four factors that residual energy, number of neighbor nodes, average distance to the neighbor nodes, distance to the center of gradient; Furthermore, it utilizes AHP to identify weighted coefficients which better reflects the impact of their proportion on object realization. Simulation results show that op-ETBG can balance load pressure of the cluster head effectively and prolong the network lifetime, suitable for large networks. In the future we will do further research on the maintaining and update of network.

\section{ACKNOWLEDGMENT}

The authors would like to thank the anonymous reviewers for the helpful comments and suggestions.

\section{REFERENCES}

[1] Jamal N.Al-karaki, Ahmal, "Routing Techniques in Wireless Sensor Networks: A Survey[J]," IEEE Wireless Communication, 2004, pp. 6-28.

[2] Akkaya K, Younis M, "a Survey on Routing Protocols for Wireless Sensor Networks[J],” Ad Hoc Networks, 2005, pp. 325-349.

[3] W.B.Heinzelman, A.P.Chandrakasan, H.Balakrishnan, "an Application-specific Protocol Architecture for Wireless Microsensor Network[J]," Wireless Communications, 2002, pp. 660-670.

[4] Lindsey S,Raghavendra C.S.PEGASIS, "Power-Efficient Gathering in Sensor Information Systems[C]," Science, vol. 3, 2002, pp. 21272130 .

[5] Na An, Xinfang Yan, Yufang Zhu, Lei Duan, "A Virtual Backbone Network Algorithm Based on the Multilevel Cluster Tree with Gateway for Wireless Sensor Networks[C]," Shanghai, December 2007 , pp. 462-465.

[6] Xinfang Yan, Lei Duan, Teng Li, "gradient-based topology control algorithm in wireless sensor networks[J]," Computer Engineering and Applications, 2011, pp. 95-98.

[7] Xinfang Yan, Zhilong Wang, Xinsheng Yan, Han Zhang, "Maintenance and update of the topology control algorithm based on gradient field in WSN[J]," Sensors and micro-systems,, 2011, pp. $56-58$.

[8] T.L.Saaty, "The Analytic Hierarchy Process[J]," New York, 1980, pp. 180-230

[9] E.Y.Kwang,C.C.Youn, "Analytic Hierarchy Process Approach for Identifying Relative Importance of Factors to Improve Passenger Security Checks at Airports[J]," Air Transport Management, 2006, pp. $135-142$.

[10] Qiangfeng Jiang,Manivannan D, "Routing protocols for sensor networks[A] Routing protocols for sensor networks[A]," Proc. IEEE Symp. Consumer Communications and Networking Conference (CCNC 04), 2007, pp. 93-98 